\title{
Propuesta de diseño de planta para incrementar la capacidad de atención de pacientes en la sala de tratamiento de hemodiálisis, Hospital III ESSALUD, Chimbote -2017
}

\section{Proposal of plant design to increase the capacity of attention for patients in room of hemodialysis treatment of the Hospital III ESSALUD - Chimbote 2017}

Luis Alejandro Quispe Carbajal ${ }^{(1)}$; Robert Fabián Guevara Chinchayan ${ }^{(2)}$; Lourdes Jossefine Esquivel Paredes ${ }^{(2)}$. Fecha de recepción: 21 de noviembre de 2017 Fecha de aprobación: 15 de mayo de 2018

\section{RESUMEN}

El presente estudio de investigación tuvo como objetivo principal la propuesta del diseño de planta para incrementar la capacidad de atención de los pacientes en la sala de tratamiento de hemodiálisis del Hospital III EsSalud - Chimbote 2017, enmarcado en un diseño de investigación no experimental, en la clasificación longitudinal. En base a los registros históricos reportados por el Hospital III EsSalud; aplicado del check-list (Resolución Ministerial $N^{\circ}$ 845-2007) y la metodología del planeamiento sistemático de distribución (SLP). El análisis evidenció que la actual sala de hemodiálisis generó pérdidas económicas mensuales de 13830 soles, además, la demanda a cubrir fue 87 pacientes, entonces, la propuesta permitió atender 112 pacientes (1344 sesiones/mes), que contó con 14 camas, atendiendo cuatro turnos de cuatro horas, estando bajo control y cumpliendo las especificaciones según el índice de capacidad que arrojó un puntaje de 3.1 (mayor al valor mínimo aceptable de 1.33). Actualmente, se logró cumplir el $50 \%$ de requisitos especificados en la resolución ministerial $\mathrm{N}^{\circ} 845$ 2007, atendiendo a 78 pacientes/semana (1093 sesiones/mes). Entonces, se determinó que la ubicación más óptima de la propuesta fue en archivos de historias clínicas, donde se distribuyó 11 departamentos necesarias para el servicio de hemodiálisis con área total de $268.9 \mathrm{~m} 2$, y lo mínimo requerido fue de $195 \mathrm{~m} 2$, además el indicador beneficio/costo resultó 1.2, siendo aceptable el estudio. En conclusión, la propuesta de diseño de planta (sala de tratamiento) pudo incrementar la capacidad de atención de pacientes de 78 a 112.

\section{PALABRAS CLAVES: Capacidad instalada, EsSalud, hemodiálisis y sistema SLP}

\section{ABSTRACT:}

This research study has as main objective, the proposal of plant design to increase the capacity of attention for patients in room of hemodialysis treatment of the Hospital III ESSALUD - Chimbote 2017, framed in a design of Non-experimental research, longitudinal classification. Based on historical records reported by the Hospital III EsSalud, applied the check-list (Resolution Ministerial $\mathrm{N}^{\circ} 845$ 2007), and the methodology of systematic layout planning (SLP). The analysis showed that current hemodialysis room generated economic losses of 13830 soles, in addition, to meet demand to cover was 87 patients, then, the proposal allowed to care for 112 patients (1344 sessions/month), which featured 14-bed hemodialysis, attending four shifts of four hours, being under control and meeting the specifications according to the capacity index that yielded a score of 3.1 (greater acceptable minimum value of 1.33 ) Currently only meets $50 \%$ of the requirements specified in the Resolution Ministerial $\mathrm{N}^{\circ}$ 845-2007, attending 78 patients / week (1093 sessions / month). Then, was determinate to the optimal location of the proposed was in archives of clinical records, where distributed 11 departments necessary to the service of hemodialysis treatment with an total area $268.9 \mathrm{~m} 2$, and the minimum required was $195 \mathrm{~m} 2$, in addition the indicator benefit/cost was 1.2 , being acceptable the study. In conclusion, the proposal of design of plant (treatment room) may increase the capacity of attention of patients from 78 to 112 .

KEY WORDS: EsSalud, Hemodialysis, Installed capacity and system SLP

\footnotetext{
(1) Autor corresponsal: Luis Quispe; Universidad César Vallejo - Filial Chimbote; Facultad de Ingeniería, Email: luis.quispe.alex@gmail.com

(2) Universidad César Vallejo- Filial Chimbote, Perú; Facultad de Ingeniería.
} 


\section{INTRODUCCIÓN}

La salud es lo primordial en el ser humano, por tal motivo, un centro hospitalario público o privado debe brindar un servicio de calidad al ciudadano, lo cual conlleva a poseer equipamiento médico de última tecnología para lograr atender a la demanda existente y por existir, portadores de una enfermedad determinada. Sin embargo, en los últimos años, se ha podido apreciar que el margen entre la oferta y la demanda, es abismal. Tal es el caso, sobre la enfermedad renal crónica (ERC) en el mundo, mostrado por el Global burden of disease study- GBD: 2013 (2015), expuso que la ERC, como la causa de muerte en la población general, ascendió del puesto 36 al 19 en tan solo dos décadas. González, Pecoits y Rosa (2013) en el registro latinoamericano de diálisis y trasplante, expresa que en 20 países de Latinoamérica, hubo un total de 358978 pacientes en terapia de remplazo renal - TRR (2010), de los cuales 224764 pacientes representan a tratamiento de hemodiálisis. Además, muestra la incidencia de la enfermedad renal en Japón y EE.UU. de 150 a 200 mil pacientes por millón de habitantes. Siguiendo con lo expuesto, el Ministerio de Salud del Perú, estimó para el año 2015, que cerca de 2507121 personas tendrían ERC en estadio pre diálisis, 19197 personas tendrían ERC terminal y la brecha no atendida sería de 7778 pacientes en el SIS (Seguro Integral de Salud). ESSALUD: Principales indicadores de salud (1990-2015), muestra gráficamente la cantidad de sesiones de Hemodiálisis que son atendidos directamente en sus instalaciones, alrededor del 2015 fueron 1'261,259 sesiones de las cuales hechas en EsSalud fueron 309,547 y en terceros fueron 951 712, mostrando así que la capacidad instalada en los Hospitales de EsSalud a nivel nacional no logra cubrir todo.

Hasta la actualidad, el Hospital III EsSalud de Chimbote, cuenta con un módulo de tratamiento de hemodiálisis, compuesta por 12 camas (10 en la sala de Hemodiálisis y dos en el cuarto de emergencia). Según el historial clínico de pacientes atendidos a partir de enero del 2015 hasta el mes de abril del 2017, en promedio se atienden a 78 pacientes por semana y en total se realizan 1093 sesiones al mes, lo que genera que cada paciente sea atendido tres veces al mes. Asimismo, las instalaciones donde esta puesta la sala de tratamiento, no está en su estado óptimo, según las observaciones directas, hay un tanque de alimentación de agua, que está en estado crítico. Otro aspecto que se dio, fue en el 2016 donde se realizó una modificación de la distribución de tubería de agua pura que se da, desde la sala de Tratamiento de agua a la Sala de Hemodiálisis y a la sala de reúsos (Hemodiálisis de emergencia), ya que estas tuberías involucradas debieron de ser instaladas por la parte interior del edificio médico, según el análisis meticulosos que se dio por las auditorias anuales a los centros médicos. Igualmente, el estado del techo en esta sala de tratamiento de agua pura, esta con agujeros por casi toda el área del techo, a la vez, la pintura se está desprendiendo poco a poco, por motivos climáticos que se han generado en este año (2017), dando a relucir la mala calidad de la composición de tarrajeo en el techo. Por otro lado, en el aspecto eléctrico, la distribución de los cables de alimentación están desordenados, e instalados a través de canaletas al exterior del techo y paredes, además, se tuvo conocimiento de sucesos respecto a desprendimientos de bloques de vidrio de la Sala de Hemodiálisis, a través de un informe generado por la Unidad renal dirigida al departamento de Mantenimiento, ocurrido el 23 de octubre del 2016. Incluso, se está generando pérdidas económicas, ya que el costo actual por sesión es de 256.65 soles, y el máximo costo por sesión según tarifario del Hospital III EsSalud es de 240 soles, entonces esto genera un costo mensual adicional de 13830 soles.

Con respecto a la distribución que presenta la actual Unidad Renal, según la Resolución Ministerial $\mathrm{N}^{\circ}$ 845-2007/MINSA, hace falta de algunos departamentos como una sala de mantenimiento de máquinas, con área mínima a los cuatro metros cuadrados, y no posee un almacén de materiales, por insuficiencia de área, ya que como mínimo este departamento en cuestión debe tener los tres metros cuadrados por modulo sin el área de cebado. En estos últimos meses, desde Abril; ha suscitado un problema mucho mayor; como ya se expresó anteriormente, al no tener una alta capacidad de atención, el Hospital III EsSalud, terciaria sus servicios de hemodiálisis en clínicas privadas; sin embargo, una de las dos clínicas, fue observada por el departamento de Nefrología del Perú, dando como conclusión el término del contrato, respecto al servicio, por lo tanto, la brecha desatendida se explayo a 87 pacientes más. 
Por tales motivos expuestos anteriormente, se hace necesario mejorar la Sala de Tratamiento y sus derivados, tanto en el nivel de servicio, incremento de la capacidad de atención instalada con una buena calidad, como en la infraestructura (mejor distribución). Entonces, se obtuvo la formulación del problema a tratar, que se expresa en la siguiente pregunta: ¿Cuál es el diseño de planta de la sala de tratamiento de hemodiálisis del Hospital III EsSalud - Chimbote 2017 que incremente la capacidad de atención de pacientes?

A ello, se le atribuye el objetivo general de estudio: Elaborar la propuesta de diseño de planta para incrementar la capacidad de atención de los pacientes de la Sala de Tratamiento de Hemodiálisis del Hospital III EsSalud - Chimbote 2017

Y los objetivos específicos fueron:

- Determinar en qué medida la recopilación estadística del crecimiento poblacional, influye en la capacidad de atención de pacientes con ERC en el Hospital III Es Salud - Chimbote 2017.

- Determinar en qué medida el diagnóstico de la actual distribución física influye en la capacidad de atención de pacientes con ERC en el Hospital III Es Salud - Chimbote 2017.

- Determinar en qué medida la nueva localización y distribución física influye en la capacidad de atención de pacientes con ERC en el Hospital III Es Salud - Chimbote 2017.

- Determinar en qué medida la evaluación beneficio/costo, influye en la capacidad de atención de pacientes con ERC en el Hospital III Es Salud - Chimbote 2017.

Por el cual, se encontraron estudios anteriores sobre este tema de estudio, como es el caso del Hospital Semma Santiago (2016), en el proyecto titulado: "Construcción y adecuación física de la unidad de servicios de hemodiálisis en el Hospital Semma Santiago". (Chile), que tiene como objetivo de construir y readecuar la unidad de servicios de hemodiálisis, bajo los requerimientos mínimos exigidos por el Ministerio de Salud Pública y Asistencial Social, a fin de proveer a los usuarios calidad y confort en los servicios. El proyecto fue establecido bajo normas estatales de Chile, por lo cual el diseño de planta que se propuso tiene los espacios físicos conforme a los puntos especificados en tales resoluciones ministeriales de Salud. Por lo cual, según los resultados de la distribución física, muestra que el espacio total brindado para la unidad de hemodiálisis, es lo suficiente para abarcar todas las áreas requeridas para brindar su servicio a los pacientes asegurados en Chile. En conclusión, el proyecto de inversión tiene un costo beneficio aceptable, ya que proporciona bienestar social y se ve reflejado en sus costos e ingresos de la nueva Unidad de servicio de Hemodiálisis.

Calderón, Zanabria y Mosquera (2015) en su tesis de grado titulada: "Creación de una clínica de hemodiálisis en la ciudad de Babahoyo, provincia Los Ríos", tiene como objetivo general el poder examinar a través del estudio técnico y financiero, la implementación de una nueva clínica de servicios de hemodiálisis en la ciudad de Babahoyo para el beneficio de la población afectada por IRC, y los cantones aledaños. El investigador ha optado por una metodología de planificación para desarrollo de un plan de negocios para apertura de una clínica de hemodiálisis. Además, expone que la capacidad instalada en la clínica (propuesta) es de 126 pacientes de hemodiálisis, resultado de las 21 máquinas, dando así que el precio se encuentre regulados por el Ministerio de Salud Pública y se ubicaría en USD 1456 para hemodiálisis. En la investigación de mercado conducida a 30 pacientes de la localidad, se pudo comprobar que existe una demanda insatisfecha. En el estudio financiero, la inversión total para la creación de la clínica se estimó en USD 377 135. Esto se realizó a un periodo de proyección de 5 años (2016-2020), que en conclusión, la clínica generará márgenes brutos, operativos y netos positivos a los largo de dicho periodo. Demostrados en la evaluación financiera del proyecto, se obtuvo un VAN de USD 506406.12 y TIR del 99.69\%. Además, en el análisis de sensibilidad, el proyecto siguió siendo rentable.

También, se pudo encontrar el estudio realizado por Zambrano (2014), en su tesis de grado titulada: "Estudio de factibilidad para la creación de un centro clínico, especializado en hemodiálisis, Cantón el Empalme, 2014".

Comienza a exponer, el investigador, que en los últimos años, se ha observado que en el mundo ha ido creciendo en forma alarmante la búsqueda de servicios de hemodiálisis para beneficios de personas 
con problemas renales, por lo tanto, el objetivo de estudio es realizar un estudio de factibilidad respecto a la creación de un centro clínico especializado en hemodiálisis, tomando en cuenta que en El Cantón Empalme (lugar de estudio), es urgente la necesidad de contar con este tipo de centro médico. El método de investigación aplicado es analítico, deductivo e inductivo. Además, se utilizó en las técnicas o instrumento de investigación a la conversación directa, las encuestas. Para llevar a cabo el estudio se tomó una muestra de 300 personas que se realizan a diario tratamientos de diálisis y residen en esta zona de estudio. El investigador llegó a la conclusión según su estudio de mercado que 155 pacientes (92\%) consideran que es de suma importancia la creación de un centro de hemodiálisis y según su evaluación financiera el TMAR (Tasa mínima aceptable de rendimiento) es de $17.68 \%$ que es el rendimiento que desean obtener los accionistas y prestadores. El resultado del VAN fue de \$235 087,08, la TIR de 39,65\% y la relación de Beneficio Costo (B/C) de $\$ 4459$ 846,92, demostrando rentabilidad a este proyecto de investigación.

Entonces, se justifica este estudio de investigación, en el entorno social, ya que la población peruana, ha ido incrementando exponencialmente durante los últimos años, de la misma manera los pacientes que padecen de enfermedades al riñón, quienes periódicamente deben de recurrir a sesiones de tratamiento de Hemodiálisis, para contrarrestar que la enfermedad llegue a un Estadio IV (Estado crítico). Como se explicó anteriormente que según EsSalud: Principales indicadores de salud (19902015), donde muestra gráficamente la cantidad de sesiones de hemodiálisis a nivel nacional que son atendidos directamente en sus instalaciones, alrededor del 2015 fueron 1261259 sesiones de las cuales hechas en Es salud fueron 309547 y en terceros fueron 951 712, mostrando así que la capacidad instalada en los Hospitales de EsSalud no logra cubrir toda esta demanda (hasta la actualidad). Actualmente, en la región Ancash, específicamente en el Hospital III EsSalud de Chimbote, la sala de tratamiento de hemodiálisis, compuesta por 12 camas (10 en la sala de hemodiálisis y 2 en el cuarto de emergencia). Según su historial clínico de pacientes atendidos a partir de enero del 2015 hasta el mes de abril del 2017 , en promedio se logra atender a 78 pacientes por semana, y que al mes, en general seis pacientes son registrados, 6 son devueltos de las clínicas para seguir con su tratamiento en Es Salud, dos nuevos, 8 trasladados a clínicas privadas, cinco fallecen y dos son recuperados por función renal. En total se realizan 1093 sesiones al mes, lo que genera que cada paciente sea atendido tres veces al mes, dentro de los turnos (cuatro) previstos por EsSalud para esta área médica. Al analizar teóricamente cuanto debería de atender la Sala de Hemodiálisis, compuesta de 12 camas de tratamiento, al servicio del ciudadano, el resultado es de 96 pacientes por semana, llevando así que el $18,75 \%$ de la capacidad instalada o no se está registrando como servicio para pacientes nuevos o se está utilizando para algunos pacientes que demandan de un lapso de tiempo mayor a lo normal, por motivos de complicaciones en el proceso del tratamiento, lo que genera que otro paciente no sea programado para una sesión más al mes. Lo antes mencionado fue constatado por las enfermeras (tres) especialistas en el tema, quienes dijeron que ese porcentaje de diferencia es tomada por pacientes en estado crítico que tomaban dos turnos. Entonces me da a entender que la capacidad instalada en la Sala de Hemodiálisis no fue tomada con restricciones ante hechos como lo ocurrido, es decir, no se hizo un análisis a través del índice de capacidad para un diseño de producto o servicio. Cabe decir, que para costear sesiones de hemodiálisis en centros médicos privados son demasiados costosos (170 dólares/sesión), conllevando a que las familiares de mediana y baja clase (mayoría de ancashinos) estén a la espera de una cita médica pública (240 soles/sesión), por meses. Ante esto, en el entorno político, el gestionar del Estado Peruano, está progresando de poco a poco, por las acciones que están tomando ante la problemática nacional de la Salud en todos los campos de enfermedades, y en nuestro caso de estudio, observando los resultados de análisis anuales que realiza el MINSA, se puede verificar el trabajo continuo de nuestro Gobierno para atender de a poco a los ciudadanos que padecen de la enfermedad renal crónica y sus derivados. Por tales motivos, me es indispensable no dejar pasar esta problemática que atenta a la Salud Ciudadana, y como profesional en Ingeniería Industrial, me es de suma importancia de dar una propuesta de mejora para reducir este índice de pacientes desatendidos en tratamientos de hemodiálisis y que sus sesiones sean más continuas.

Por ello, se hace el estudio de un diseño de planta para incrementar la capacidad de atención a pacientes de la sala de tratamiento de hemodiálisis del Hospital III EsSalud- Chimbote. 


\section{MATERIAL Y MÉTODOS}

El diseño de investigación realizado en el Hospital III - Es Salud de Chimbote fue No Experimental, en la clasificación Longitudinal, debido a que no hay manipulación intencional de la variable de estudio, y el análisis a través del tiempo estuvo inmerso en el costo por sesión que generó la propuesta de diseño de planta (sala de tratamiento de hemodiálisis).

Por ende, se elaboró el cuadro de operacionalización según la variable de estudio, especificado en la tabla 01.

Tabla 01: Cuadro de operacionalización de variable de estudio

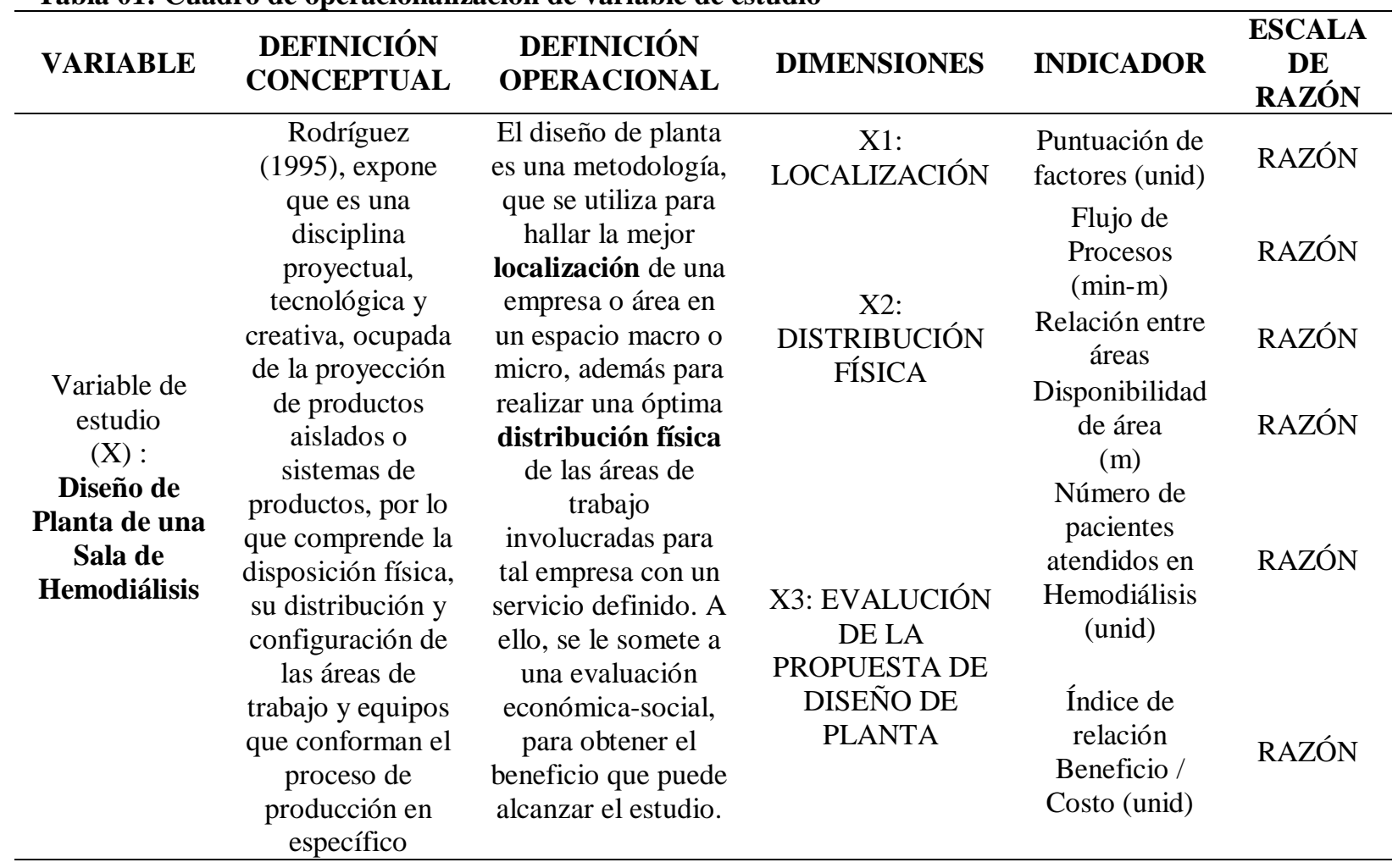

En este estudio no se aplicó ninguna encuesta por lo tanto no se calculó ninguna muestra, ni se obtuvo ninguna población, pero se pudo aplicar técnicas como la revisión documental, observación directa, organización y métodos, por último el análisis de datos, para ello se utilizó los siguientes instrumentos de recolección de datos como el registro de datos, para obtener la cantidad de demanda a satisfacer y el número de pacientes atendidos actual, el check-list, para conocer el porcentaje de cumplimiento respecto a la infraestructura en la actual sala de hemodiálisis, registro de herramientas del planeamiento sistémico de distribución, con el que se obtuvo la propuesta de diseño de planta de la sala de hemodiálisis que incremente la capacidad de atención de pacientes, por último, registro de costos, para la evaluación de la propuesta, a través del indicador de beneficio/costo.

\section{Método de Análisis}

Se utilizó la estadística descriptiva, hallando el promedio o media de pacientes con ERC en la región de Ancash-Chimbote, a través de datos históricos del INEI del Perú, para así proyectar la demanda de pacientes con ERC que habrá dentro de 10 años, y determinar la capacidad instalada más adecuada para suplir la demanda insatisfecha a través del índice de capacidad. Por ello la aplicación del instrumento de registro de datos, el cual fue procesado en el Microsoft Office Excel 2010. 
Una vez obtenido estos resultados, se pasó a la evaluación actual de la sala de hemodiálisis respecto a su diseño de planta, a través de un Check-list desarrollado por ítems que son puntualizados por la Resolución Ministerial N ${ }^{\circ} 845-2007$, lo cual nos proporcionó qué aspectos deben ser considerados en la propuesta de diseño de planta, este Check-list fue procesado en el Microsoft Office Excel 2010. Ya teniendo estos dos resultados de evaluación, se pasó a la implementación del análisis de Capacidad Instalada, que nos arrojó la cantidad de pacientes con ERC que serán atendidos, y las herramientas del planeamiento sistemático de distribución, que nos proporcionó la mejor opción de distribución de los departamentos involucrados en la atención de la sala de hemodiálisis, al igual que los anteriores puntos, estos análisis fueron desarrollados en el Microsoft Office Excel 2010.

Por último, se logró hallar la relación entre el beneficio brindado con la propuesta de diseño de planta con respecto a los costos involucrados en el mejorado servicio, tal análisis fue procesado en el Microsoft Office Excel 2010.

\section{RESULTADOS}

Primer objetivo específico se basó en el diagnóstico situacional de la empresa respecto a la capacidad instalada. Para ello, se aplicó la estadística descriptiva, haciendo un análisis de la demanda y oferta del servicio de hemodiálisis en el Perú, por medio de datos obtenidos del Instituto Nacional de Estadística e Informática - INEI, donde se logró encontrar la cantidad total de pacientes en tratamiento de hemodiálisis (11195 pacientes) de los cuales la región Ancash equivale el $2 \%$ del total, donde se divide en dos Sedes, Chimbote con un total de $\mathbf{7 8}$ pacientes en Hospital III EsSalud y Huaraz con 32 pacientes, la diferencia están en clínicas particulares ubicadas en Nuevo Chimbote ( $\mathbf{8 7}$ pacientes que son la demanda insatisfecha actual). No obstante, se comprobó a través del punto de equilibrio que la actual sala de hemodiálisis está generando pérdidas económicas al Hospital III EsSalud, ya que al mes se atienden 1093 sesiones dando así la gráfica aprecia en la Tabla 01 y la cantidad del PE en Fig. 02

Tabla 02: Cantidad de sesiones según el punto de equilibrio

\begin{tabular}{|c|c|c|}
\hline $\begin{array}{c}\text { PUNTO } \\
\text { EQUILIBRIO }\end{array}$ & COSTO FIJO & COSTO VARIABLE \\
\hline 1189 Sesiones/mes & 171710.30 & 95.55 \\
\hline 99 pacientes/semana & \multicolumn{2}{|}{} \\
\cline { 1 - 2 }
\end{tabular}

Fuente: Elaboración Propia basado en Yermanos y Correa (2011)

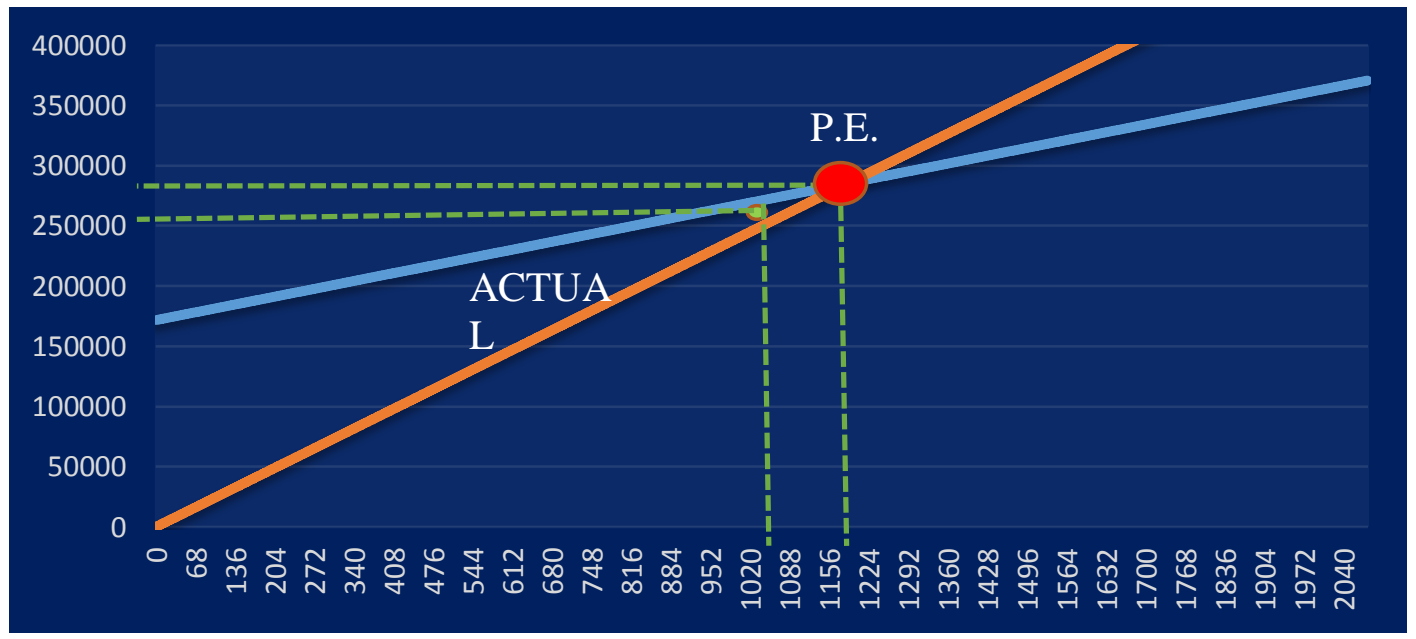

Fig.01: Gráfica del punto de equilibrio

Además, se sometió a un análisis estadístico de control, a través del índice de capacidad (Cpk - Tabla 03), para evidenciar, si cumple con las especificaciones requeridas o no. Entonces, la actual sala de hemodiálisis, arroja un 0.443 , siendo menor al valor mínimo aceptable (1.33), no cumple con las especificaciones deseadas, sin embargo con la nueva capacidad proyectada, el valor arrojado es de 3.1, mayor al valor mínimo del Cpk, mostrando así que el proceso estará bajo control y cumplirá con las 
condiciones deseadas. Estos se pueden apreciar en la Fig.02 con más exactitud la diferencia entre ambas capacidades.

Tabla 03: Índice de capacidad actual vs mejora

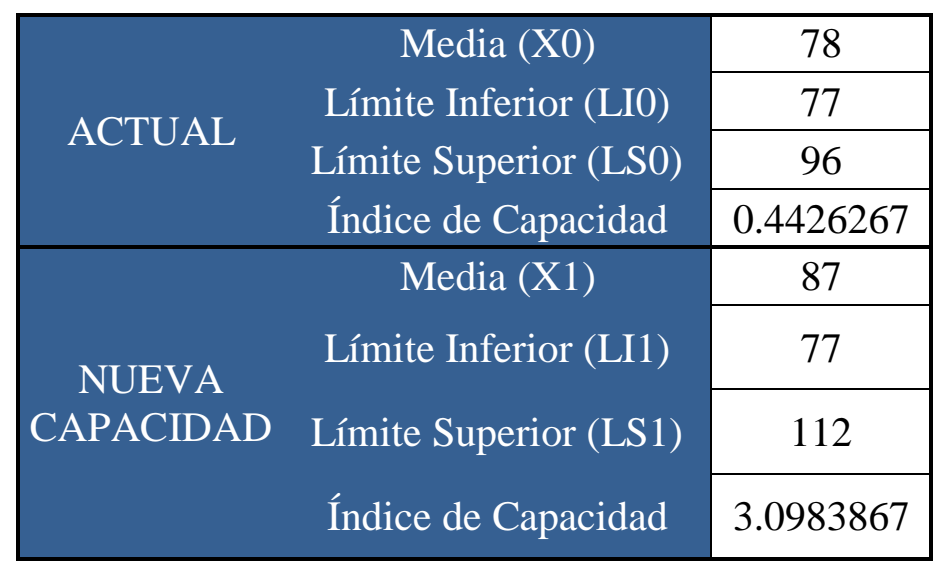

Fuente: Elaboración propia basado en Chase y Aquilano (2009)

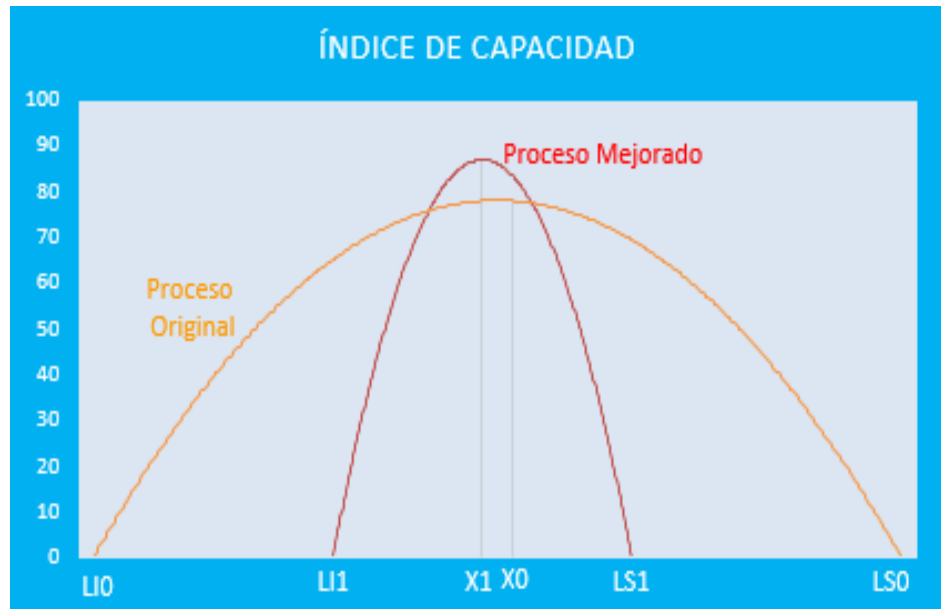

Fig.02: Gráfica del índice de capacidad actual vs mejora

Obtenido el primer objetivo específico desarrollado, se pasó al diagnóstico de la actual distribución física. En el que se aplicó el Check-List: "Instalación de una sala de Hemodiálisis para servicios de hemodiálisis básica del establecimiento de salud". Este instrumento, fue validado por la Resolución Ministerial $N^{\circ} 845-2007$ del Perú, donde aclara todos los puntos que deben de respetar para brindar un servicio sólido de Hemodiálisis, que conlleva a que el paciente salga satisfecho con este servicio público. Para ello, los resultados se evidencian en las Tablas 04 y 05 que representan los resúmenes cuantitativos de cumplimiento en general.

Tabla 04: Resumen general de Check-list

\begin{tabular}{|c|c|c|c|}
\hline CHECK - LIST DE INSTALACIÓN DE UNA SALA DE HEMODIÁLISIS PARA SER VICIOS DE \\
HEMODIÁLISIS BÁSICA DEL ESTABLECIMIENTO DE SALUD \\
\hline GUÍA SEGÚN LA RESOLUCIÓN MINISTERIAL N ${ }^{\circ} 845-2007$ \\
\hline REQUISITOS & CUMPLIMIENTO & \multirow{2}{*}{$\%$ DE CUMPLIMIENTO } \\
\cline { 2 - 4 } & SI & NO & \\
\hline ORGANIZACIÓN Y FUNCIONAMIENTO & 2 & 0 & $100 \%$ \\
\hline ATENCIÓN DEL PACIENTE & 1 & 0 & $100 \%$ \\
\hline BIOSEGURIDAD & 4 & 1 & $80 \%$ \\
\hline
\end{tabular}




\begin{tabular}{|c|c|c|c|}
\hline RECURSOS HUMANOS & 4 & 3 & $57 \%$ \\
\hline INFRAESTRUCTURA & 12 & 19 & $39 \%$ \\
\hline TOTAL & 23 & 23 & $50 \%$ \\
\hline
\end{tabular}

Tabla 05: Resumen de requisito con menor cumplimiento

\begin{tabular}{|c|c|c|c|}
\hline \multirow{2}{*}{ REQUISITOS } & \multicolumn{2}{|c|}{ CUMPLIMIENTO } & \multirow{2}{*}{ \% DE CUMPLIMIENTO } \\
\hline & SI & NO & \\
\hline INFRAESTRUCTURA & 12 & 19 & $39 \%$ \\
\hline ESPECIFICACIONES GENERALES & 6 & 13 & $32 \%$ \\
\hline SALA DE TRATAMIENTO DE AGUA & 3 & 1 & $75 \%$ \\
\hline SALA DE HEMODIÁLISIS & 3 & 2 & $60 \%$ \\
\hline SALA DE CEBADO Y ALMACENAMIENTO & 0 & 3 & $0 \%$ \\
\hline
\end{tabular}

El porcentaje de cumplimiento actual de la Sala de Hemodiálisis es de $50 \%$ en general, además se aprecia que el requisito correspondiente a Recursos Humanos posee un cumplimiento del $100 \%$, reflejando que está bien coordinado. Por último, Se estratificó el aspecto con menos cumplimiento que es Infraestructura con un 39\% de cumplimiento, evidenciando que se necesita con urgencia una nueva sala de hemodiálisis y un posible mantenimiento general a la actual, para que esté acta para atender a la demanda actual de 78 pacientes, y sea como complemento de la nueva sala a diseñar.

Además, se recopiló la cantidad de pacientes atendidos en la actual sala de Hemodiálisis, estos datos obtenidos fueron de la base de datos del departamento de Unidad Renal del Hospital III Es Salud, donde se obtuvo que al mes, se atienden en promedio a 312 pacientes en la sala de Hemodiálisis actual, con un promedio general de 1093 sesiones, y el dato importante de estudio es que a la semana se logran atender a 78 pacientes $(81,25 \%$ de la cap. teórica), ahora contrastando con la capacidad teórica, se aprecia que solo se alcanzó utilizar el $95 \%$ de las sesiones que debería haber obtenido.

Obtenido el diagnóstico de la actual sala se pasó a obtener la propuesta del diseño de planta empezando por el:

Cálculo de la Capacidad Teórica propuesta: La nueva capacidad ha sido calculada sistemáticamente en Excel, solo se pide los turnos que serán programados y la cantidad de camas propuestas, proporcionándonos la capacidad propuesta equivalente a $\mathbf{1 1 2}$ pacientes por semana a atender, contribuyendo a un aumento del $14.3 \%$ de la capacidad teórica actual (96pacientes/semana), esto es al incremento de camas, ya que el espacio métrico es lo suficiente para albergar esta cantidad, y queda demostrado que es más que suficiente para lograr atender a la demanda proyectada promedio (87pacientes/semana).

Luego la elección de la mejor ubicación para la Sala de Hemodiálisis donde se aplicó el método de factores por puntuación para elegir la mejor ubicación de la sala de Hemodiálisis, en la parte interna del Hospital III Es Salud. Como se puede observar en la Tabla 06.

Por lo tanto, el lugar más propicio, según este método de localización, es el espacio utilizado actualmente por el Archivero de Historias clínicas, ya que posee demasiada área y esta estratégicamente ubicado, esto lo refleja con la puntuación final de 62, por otro lado, no era factible la expansión de la sala de hemodiálisis actual, ya que no tenía área suficiente de expansión, además que quitaba espacio al estacionamiento del Hospital III Es Salud, que posee una infraestructura con elevación de piso.

Tabla 06: Nueva ubicación de la sala según Método de puntuación de factores

\begin{tabular}{|c|c|c|c|c|c|}
\hline \multirow{2}{*}{ FACTOR } & PESO & \multicolumn{2}{|c|}{ ARC. HISTORIA CLINICAS } & \multicolumn{2}{|c|}{ EXPANSION EN LA ACTUAL } \\
\cline { 2 - 6 } & $\%$ & CALIFICACION & PONDERACION & CALIFICACION & PONDERACION \\
\hline $\begin{array}{c}\text { CERCANIA DE LAS } \\
\text { PUERTAS DE } \\
\text { INGRESO }\end{array}$ & 0.15 & 8 & 1.2 & 7 & 1.05 \\
\hline
\end{tabular}




\begin{tabular}{|c|c|c|c|c|c|}
\hline $\begin{array}{c}\text { CERCANIA DE LOS } \\
\text { INSUMOS }\end{array}$ & 0.2 & 10 & 2 & 8 & 1.6 \\
\hline $\begin{array}{c}\text { INFRAESTRUCTURA } \\
\text { DISPONIBLE }\end{array}$ & 0.3 & 10 & 3 & 5 & 1.5 \\
\hline $\begin{array}{c}\text { DISPONIBILIDAD } \\
\text { DE RED ELECTRICA }\end{array}$ & 0.12 & 9 & 1.08 & 9 & 1.08 \\
\hline $\begin{array}{c}\text { DISPONIBILIDAD } \\
\text { DE RED SANITARIA }\end{array}$ & 0.12 & 9 & 1.08 & 9 & 1.08 \\
\hline SEGURIDAD & 0.05 & 8 & 0.4 & 8 & 0.4 \\
\hline $\begin{array}{c}\text { MANO DE OBRA } \\
\text { DISPONIBLE }\end{array}$ & 0.06 & 8 & 0.48 & 8 & 0.48 \\
\hline TOTAL & 1 & 62 & 9.24 & 54 & 7.19 \\
\hline
\end{tabular}

Luego la propuesta del diseño de planta respecto a la distribución Física de la Sala de Hemodiálisis según Herramientas del Planeamiento Sistemático de Distribución -SPL

Para ello, se utilizó el Gráfico Producto - Cantidad (Gráfico P-Q), a través de esta herramienta se obtuvo la proyección de sesiones anuales que se harán durante el período 2018 al 2027, como se muestra en la Tabla 07. Y por tal motivo, se reflejó que esta cantidad proyectada está en un decrecimiento por motivos de la tasa de crecimiento, por lo que la capacidad teórica instalada para la nueva sala de hemodiálisis, está por encima de esta cantidad proyectada de sesiones, en un $25 \%$, margen aceptable.

Tabla 07: Cantidad de sesiones demandadas vs sesiones por nueva capacidad teórica

\begin{tabular}{|c|c|c|c|}
\hline \multirow{2}{*}{ AÑ } & \multirow{2}{*}{ PRODUCTO } & \multicolumn{2}{|c|}{ SESIONES ANUALES } \\
\cline { 3 - 4 } & SESIONES-2018 & $\begin{array}{c}\text { SEGÚN CANTIDAD } \\
\text { DEMANDADA }\end{array}$ & $\begin{array}{c}\text { SEGÚN CAPACIDAD } \\
\text { TEÓRICA }\end{array}$ \\
\hline 2018 & SESIONES-2019 & 13233 & 16128 \\
\hline 2019 & SESIONES-2020 & 12984 & 16128 \\
\hline 2020 & SESIONES-2021 & 12678 & 16128 \\
\hline 2021 & SESIONES-2022 & 12152 & 16128 \\
\hline 2022 & SESIONES-2023 & 11903 & 16128 \\
\hline 2023 & SESIONES-2024 & 11645 & 16128 \\
\hline 2024 & SESIONES-2025 & 11378 & 16128 \\
\hline 2025 & SESIONES-2026 & 11140 & 16128 \\
\hline 2026 & SESIONES-2027 & 10917 & 16128 \\
\hline 2027 & PROMEDIO & 12042 & $\mathbf{2 5 . 3 3 \%}$ \\
\hline DIFERENCIA & & & \\
\hline
\end{tabular}


Luego se procedió a realizar el diagrama de Proceso de Actividades - DAP, para el servicio de hemodiálisis actual, el cual mostró que en total se requiere de 4 horas por sesión, tiempo fijo programado por sesión, y que en general la distancia es de 47,81 m de recorrido, dentro de la sala de hemodiálisis. Con más detalle se observan todos los procesos en la Fig. 03.

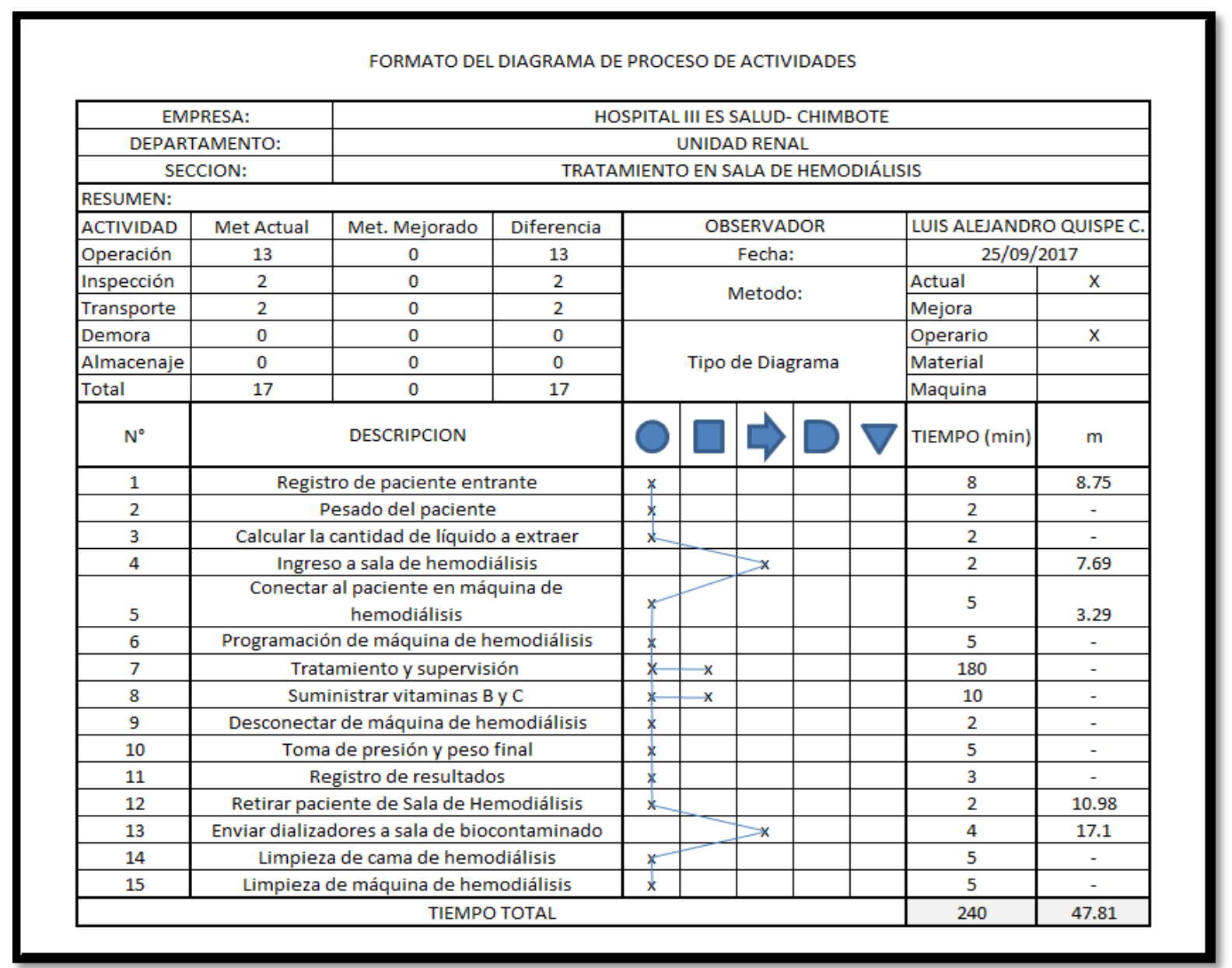

Fig.03: Diagrama de análisis de proceso del servicio de tratamiento de hemodiálisis (Escala 1/2)

Fuente: Elaboración propia Carro y Gonzales (2014)

Después se realizó la tabla relacional de Actividades, una vez realizado el diagrama de proceso de actividades, se pasó a detallar las áreas involucradas para la nueva sala de hemodiálisis, a través de la herramienta relacional de actividades, los cuales nos proporcionó cuales son las áreas imprescindibles una de otra y cuales tienen una relación indeseable por lo que se necesitará separarse una de otra. Para más detalle, se puede observar en la Tabla 08 y Tabla 09.

Tabla 08: Significado de simbología para tabla relacional de actividades

\begin{tabular}{|c|c|c|c|c|}
\hline CÓDIGO & DEFINICIÓN & & CÓDIGO & DEFINICIÓN \\
\hline $\mathrm{A}$ & ABSOLUTAMENTE NECESARIA & & 1 & \multirow{6}{*}{$\begin{array}{c}\text { SUPERVISIÓN CONTINUA } \\
\text { COMPARTEN MISMO ESPACIO } \\
\text { COMUNICACIÓN CON PERSONAL } \\
\text { SECUENCIA DEL FLUJO DE } \\
\text { TRABAJO } \\
\text { MOLESTIAS/PELIGROS }\end{array}$} \\
\hline $\mathrm{E}$ & ESPECIALMENTE IMPORTANTE & & 2 & \\
\hline I & IMPORTANTE & $\mathrm{A}$ & 3 & \\
\hline $\mathrm{O}$ & ORDINARIA & 1 & 4 & \\
\hline $\mathrm{U}$ & NO IMPORTANTE & & 5 & \\
\hline $\mathrm{X}$ & INDESEABLE & & & \\
\hline
\end{tabular}

Fuente: Elaboración propia Chase y Aquilano (2009) 
Tabla 09: Tabla relacional de actividades para sala de hemodiálisis - HD

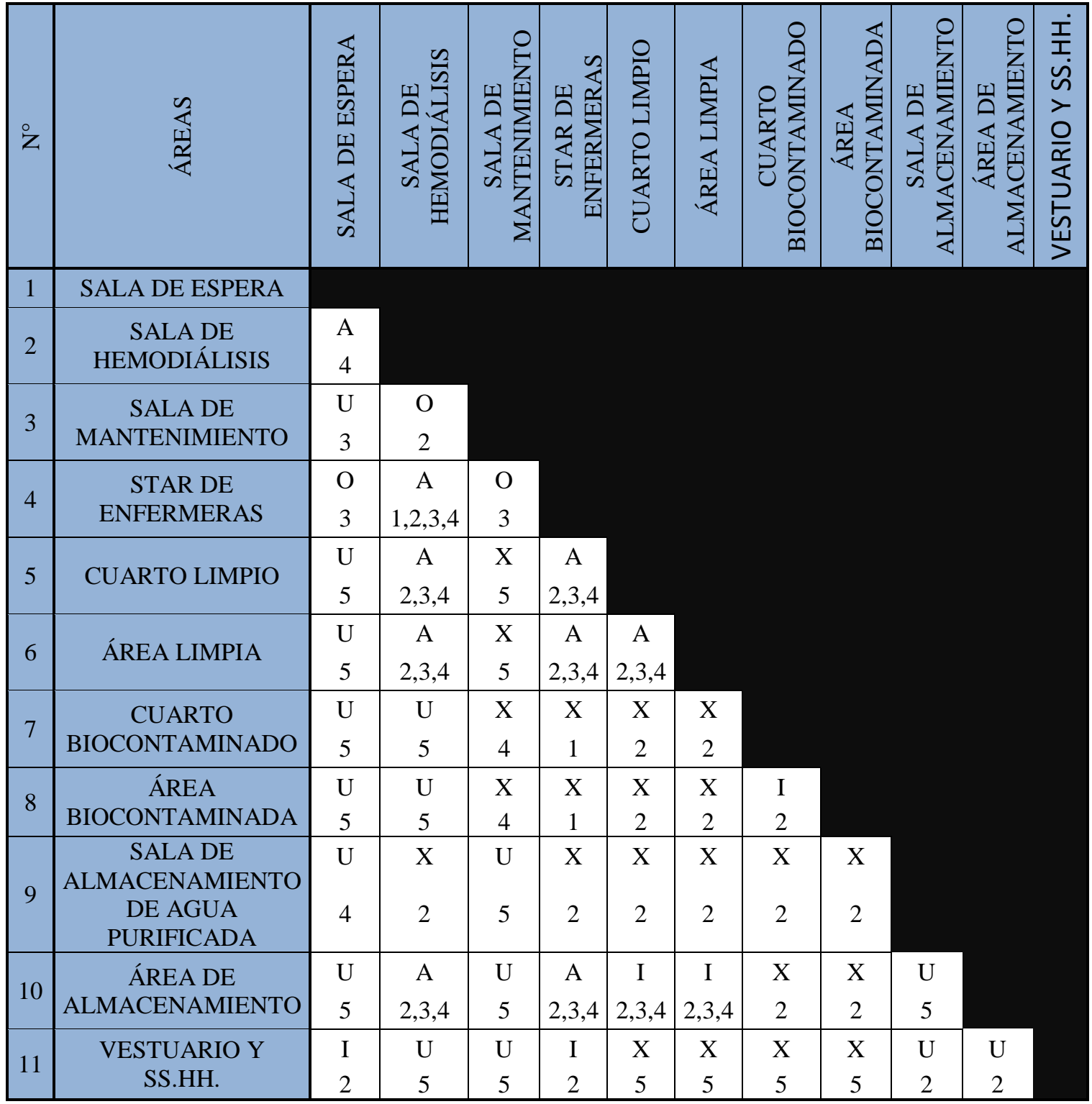

Luego se procedió a elaborar el diagrama Relacional de Actividades, por lo que se le dio un color específico para cada código, para no poder confundirse, y así se llegó a lo mostrado en la Fig. 04

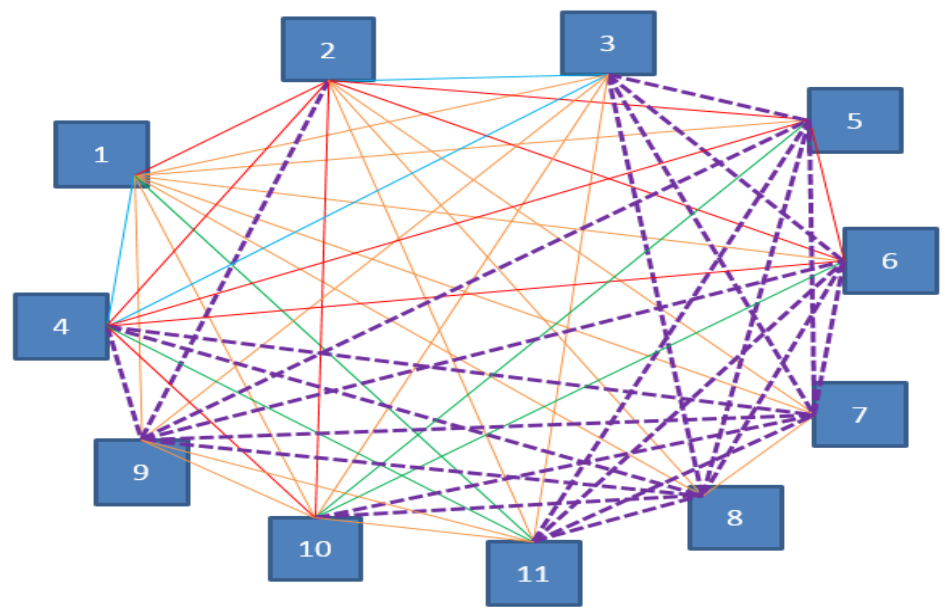

Fig.04: Diagrama relacional de actividades para sala de HD (Escala 1/2) Fuente: Elaboración propia 
Consecutivo a ello se culminó el diagrama relacional de espacios y recorrido (Fig.05), para ello, se necesitó el área de cada departamento involucrado en el servicio de hemodiálisis, por lo que, los datos fueron referenciados según la Resolución ministerial $N^{\circ}$ 845-2007, evidenciándolo resumidamente en la Fig. 09. Donde se especifica que el área total mínima requerida es de 195 metros cuadrados. Y como último paso de la metodología del SPL (Planeamiento Sistemático de la Distribución), se elaboró el LAYOUT final para la nueva sala de Hemodiálisis, como resultado de la aplicación de todas las herramientas mencionadas anteriormente; evidenciado en Fig. 07. Y como detalle se especifica en la Fig.06, los metros cuadrados utilizados por cada departamento en la nueva ubicación de la Sala de Hemodiálisis, siendo mayor a lo pedido por la R.M. 845-2007.

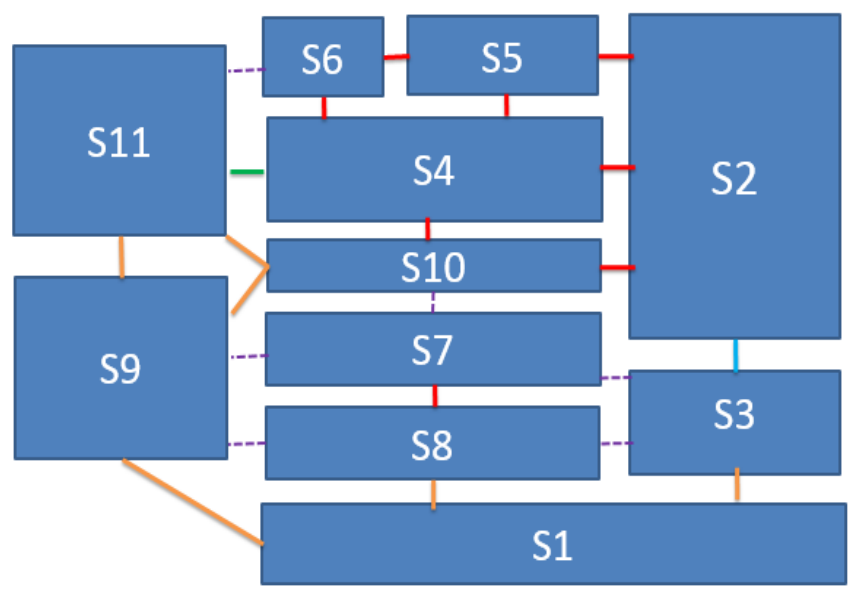

Fig.05: Diagrama relacional de espacios sala de hemodiálisis

\begin{tabular}{|c|c|c|c|}
\hline $\mathrm{N}^{\circ}$ & DEPARTAMENTOS & $\begin{array}{l}\text { ÁREA }(\mathrm{m} 2)- \\
\text { Según R.M. } \\
8.45-2007\end{array}$ & $\begin{array}{c}\text { ÁREA } \\
\text { (m2) del } \\
\text { Diseño } \\
\text { Propuesto }\end{array}$ \\
\hline s1 & SALA DE ESPERA & 30 & 49 \\
\hline $\mathrm{s} 2$ & $\begin{array}{l}\text { SALA DE } \\
\text { HEMODIÁLISIS }\end{array}$ & 116 & 154 \\
\hline $\mathrm{s3}$ & $\begin{array}{l}\text { SALA DE } \\
\text { MANTIENIMIENTO }\end{array}$ & 4 & 10.2 \\
\hline s4 & STAR DE ENFERMERAS & 8 & 9.8 \\
\hline s5 & CUARTO LIMPIO & 4 & 4.2 \\
\hline 56 & ÁREA LIMPIA & 2 & 3 \\
\hline s7 & $\begin{array}{l}\text { CUARTO } \\
\text { BIOCONTAMINADO }\end{array}$ & 4 & 4.5 \\
\hline 58 & $\begin{array}{l}\text { ÁREA } \\
\text { BIOCONTAMINADA }\end{array}$ & 4 & 5.2 \\
\hline s9 & $\begin{array}{l}\text { SALA DE } \\
\text { ALMACENAMIENTO } \\
\text { DE AGUA PURIFICADA }\end{array}$ & 10 & 10.5 \\
\hline s1o & $\begin{array}{c}\text { AREA DE } \\
\text { ALMACENAMIENTO }\end{array}$ & 3 & 3.5 \\
\hline s.11 & VESTUARIO Y SS.HH. & 10 & 15 \\
\hline & & 195 & 268.9 \\
\hline
\end{tabular}

Fig.06: Área según RM 845 vs área propuesta a utilizar (Escala $1 / 2$ )

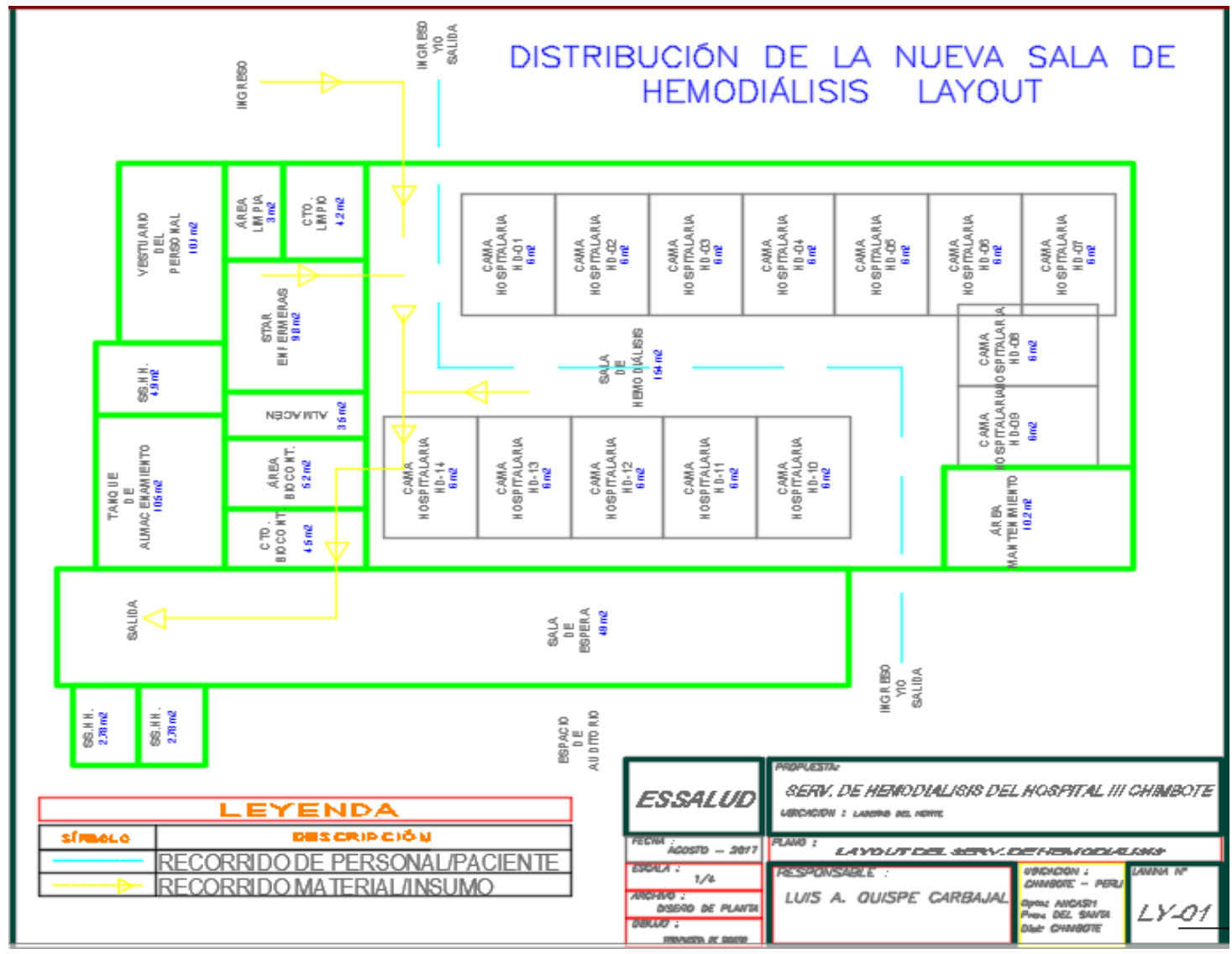

Fig.07: Layout final de sala de hemodiálisis (Escala 1/2)

Por último objetivo específico, se hizo la evaluación beneficio/costo, con la obtención de los costos del servicio de hemodiálisis por sesión, se logró obtener el beneficio/costo, como se observa en la 
Tabla 10, donde evidencia que en el periodo del 2015 al 2017, el resultado equivale a 0,95, lo que indica que no es aceptable la actual sala de hemodiálisis, ya que se han generado más costos que ingresos por el servicio, contrayendo perdidas económicas (13 830 soles mensuales) para el Hospital III Es Salud, mientras que con esta nueva sala de hemodiálisis y su capacidad propuesta, los costos van a ser reducidos (234.94 soles/sesión), llevando a que haya ganancia, y para reforzar lo dicho, en el periodo del 2018 al 2027, el indicador arroja 1,2,es decir que por cada Nuevo Sol invertido se genera un ingreso de 20 centavos, demostrando que es aceptable y rentable esta propuesta de diseño de planta de la sala de hemodiálisis.

Tabla 10: Indicador de beneficio/costo desde 2015 al 2027

\begin{tabular}{cccc}
\hline AÑOS & INGRESO & COSTO & B/C \\
\hline 2015 & 3119760 & 3289787 & 0,95 \\
2016 & 3154320 & 3326230 & 0,95 \\
2017 & 3210480 & 3385451 & 0,95 \\
2018 & 3870720 & 3218504 & 1,20 \\
2019 & 3870720 & 3218504 & 1,20 \\
2020 & 3870720 & 3218504 & 1,20 \\
2021 & 3870720 & 3218504 & 1,20 \\
2022 & 3870720 & 3218504 & 1,20 \\
2023 & 3870720 & 3218504 & 1,20 \\
2024 & 3870720 & 3218504 & 1,20 \\
2025 & 3870720 & 3218504 & 1,20 \\
2026 & 3870720 & 3218504 & 1,20 \\
2027 & 3870720 & 3218504 & 1,20 \\
\hline
\end{tabular}

\section{IV.DISCUSIÓN}

Calderón, Zanabria y Mosquera (2015) realizaron un proyecto de inversión para una Clínica de Hemodiálisis en la ciudad de Babahoyo, en la cual aplicaron un estudio de mercado, el cual les mostró que es de suma importancia la instalación de una clínica de este tipo de tratamiento, por lo que su demanda a satisfacer fue de 126 pacientes/semana, con la implementación de 21 camas de hemodiálisis, y con tres turnos laborales de ocho horas. Entonces, procesando estos datos al instrumento aplicado para la obtención de la capacidad teórica a instalar para este estudio, arrojó que la proporción cama/paciente es correcta, por lo que, valida aún más el instrumento, lo único que cambia dentro de este estudio es que se proyecta atender dentro de cuatro turnos de cuatro horas, con la implementación de 14 camas, satisfaciendo a 112 pacientes/semana.

El Hospital SEMMA SANTIAGO, realizó la construcción y adecuación física de la unidad de servicios de hemodiálisis, para lo cual tuvo como guía las especificaciones técnicas enmarcadas en su Resolución Ministerial de Salud respecto a este servicio público. Las dimensiones de áreas son muy similares a la Resolución Peruana, ya que estas normas son estandarizadas internacionalmente. Dentro de ello, nos mostró un ejemplo de distribución física completa, haciendo la comparativa con la sala de tratamiento en estudio de propuesta, que también respetó las normativas peruanas, poseen una similitud en la distribución, lo único de variante es el recorrido de los materiales e insumos, ya que la ubicación donde se establecerá a la sala de hemodiálisis, es estratégica, conectándose con el ingreso principal de pacientes, y el ingreso de emergencia, además que la salida de los insumos y/o materiales utilizados en el tratamiento son retirados instantáneamente a las áreas de materiales biocontaminados.

En los estudios de investigación de Calderón, Zanabria y Mosquera (2015) y Zambrano (2014), realizaron un estudio de inversión, aplicando sus indicadores respectivos, como son el Valor Actual Neto (VAN), Tasa de Interés de Retorno (TIR) y Beneficio/costo, proyectándolo en un periodo de estudio de 5 años, lo cual les dio cifras numéricas positivas, sobrepasando los límites que exige cada indicador para ser aprobado en proyecto. Entonces, haciendo la comparativa con esta investigación de estudio no experimental, de clasificación longitudinal, en la cual no se realizó un estudio de inversión, por motivos del diseño, solo se aplicó el indicador de Beneficio/costo, que arrojó 1.2 (mayor 
al límite que permite este indicador),es decir por cada Nuevo Sol invertido se genera un ingreso de 20 centavos, por lo que se puede proyectar y matemáticamente decir que en el caso de hacerse el estudio de inversión exhausto nos generarían un VAN Y TIR en estado positivo y mayor a su mínimo de aceptación, afirmando contundentemente que esta investigación es rentable y factible, ya que beneficioso sí lo es, además que la propuesta del diseño de planta no generó pérdidas económicas, como se evidencia actualmente ( 13830 soles al mes).

\section{CONCLUSIÓN}

Se determinó que la recopilación estadística del crecimiento poblacional, influyó en la capacidad de atención de pacientes con ERC en el Hospital III EsSalud - Chimbote 2017, y esto se hizo posible con la aplicación del punto de equilibrio, donde se apreció que la actual sala de hemodiálisis está en la zona de pérdidas (13830 soles mensuales de gastos adicionales) y a través del índice de capacidad nos arrojó que no está bajo las especificaciones exigidas, mientras que la propuesta de diseño de planta de la sala de hemodiálisis, a través de la proyección, según la tasa de crecimiento, nos dio a conocer que si está bajo control con un índice de 3,1 (mayor al mínimo equivalente a 1,33) y cumple las especificaciones exigidas para la demanda a satisfacer. Además, se obtuvo el diagnóstico de la actual distribución física, a través de la aplicación de un Check-List, basado en la Resolución Ministerial 845-2007, con la que mostró que el cumplimiento actual es del 50\%, estado inaceptable, por lo que se pudo afirmar, que la actual distribución sí influyó en la capacidad de atención de pacientes con ERC en el Hospital III Es Salud - Chimbote 2017, ya que el espacio necesario para las máquinas de hemodiálisis son menores a lo establecido por la Resolución Ministerial 845-2007, y que fueron obviados algunos departamentos para este servicio de salud. También, se determinó la nueva localización, a través del método de puntuación de factores, arrojando que la nueva Sala de Hemodiálisis estará en el espacio de archivero de Historias clínicas y la propuesta de distribución física influyó en el incremento de la capacidad de atención de pacientes con ERC en el Hospital III EsSalud - Chimbote 2017, ya que se pudo posicionar en esta área a 14 camas de hemodiálisis, con la que se pudo atender a los 112 pacientes/semana. A todo ello, se determinó la evaluación del indicador de beneficio/costo que fue equivalente a 1,2, demostrando así que, la propuesta de diseño de planta, es aceptable ya que supera la unidad, margen mínimo de aceptabilidad de este indicador.

Finalmente, se obtuvo la propuesta de diseño de planta para incrementar la capacidad de atención de los pacientes de la sala de tratamiento de hemodiálisis del Hospital III EsSalud - Chimbote 2017, a través de la metodología SLP, por lo que el incremento de la capacidad fue de 78 a 112 pacientes/semana. 


\section{REFERENCIAS}

Calderón, D.; Zanabria, X. y Mosquera, R. (2015). Creación de una clínica de hemodiálisis en la ciudad de Babahoyo, provincia los Ríos. (Tesis de Magister en Gerencia Hospitalaria). Escuela Superior Politécnica del Litoral. Escuela de Postgrado en Administración de Empresas. Guayaquil

Chase, R., Roberts, F. y Aquilani, N. (2009). Administración de operaciones: Producción y cadena de suministro (10 ed.). México: McGraw-Hill/Interamericana editores S.A.

Córdoba, M. (2011). Formulación y evaluación de proyectos (2ed). Bogotá: Eco Ediciones.

EsSalud (2016). Principales indicadores de salud 1990-2015. Recuperado de: http://www.essalud.go...b.pe/downloads/series_estadisticas_1990_2015_VF.pdf

GBD 2013. (2015). Mortality and causes of death collaborators. Global, regional, and national agesex specific all-cause and cause-specific mortality for 240 causes of death, 1990-2013: a systematic analysis for the global burden of disease study 2013. Lancet.

González, C., Pecoits, R. y Rosa, G. (2013). Registro Latinoamericano de diálisis y trasplante renal. Buenos Aires. Recuperado de: http://www.slanh.net/wpcontent/uploads/2014/07/Presentacion del RFADTR en_el_VI_Congress_of_the_Internat ional_Society_of_Hemodialysis_Buenos_Aires_2013.pdf

Hospital Semma Santiago (2016). Construcción y adecuación física de la unidad de servicios de hemodiálisis en el Hospital SEMMA Santiago. Chile: Santiago.

Ministerio de Salud. (2016). Análisis de la situación de la enfermedad renal crónica en el Perú, 2015 Jesús María: Dirección General de Epidemiologia.

Rodríguez, G. (1995). Manual de diseño industrial: curso básico (3 ed.). México: Ediciones G.Gili S.A.

Yérmanos, E. \& correa, L. (2011). Contabilidad administrativa un enfoque gerencial de costos. Santiago de Cali: U. ICESI. Recuperado de: https://repository.icesi.edu.co/biblioteca_digital/bitstream/10906/66580/1/libro_contable_a dministrativo.pdf

Zambrano, J. (2014) Estudio de factibilidad para la creación de un centro clínico, especializado en hemodiálisis, Cantón el Empalme, 2014. (Tesis de grado en Ingeniería en Gestión Empresarial). Universidad Técnica Estatal de Quevedo. Facultad de Ciencias Empresariales. Ecuador 